\title{
EVERY FINITE GROUP IS THE AUTOMORPHISM GROUP OF SOME FINITE ORTHOMODULAR LATTICE
}

\author{
GERALD SCHRAG
}

\begin{abstract}
If $L$ is a lattice, the automorphism group of $L$ is denoted $\operatorname{Aut}(L)$. It is known that given a finite abstract group $H$, there exists a finite distributive lattice $D$ such that $\operatorname{Aut}(D) \cong H$. It is also known that one cannot expect to find a finite orthocomplemented distributive (Boolean) lattice $B$ such that $\operatorname{Aut}(B) \cong H$. In this paper it is shown that there does exist a finite orthomodular lattice $L$ such that $\operatorname{Aut}(L) \cong H$.
\end{abstract}

1. Introduction. An important question in orthomodular lattice theory from the physicist's point of view is whether or not every group is the automorphism group of some orthomodular lattice. As indicated in the title of this paper, our purpose is to give an affirmative answer for the finite case. Corresponding results with cardinality bounds for lattices and distributive lattices can be found in [6] and [1], respectively. A corollary to our result is that every finite group is the automorphism group of a quantum logic. The relevance of orthomodular lattice theory to quantum mechanics can be found in [2], [10], [11], and [12].

2. Preliminary results. Prior to the development of the main result, several definitions will be reviewed.

An incidence structure is a triple $S=(\mathfrak{p}, \mathfrak{B}, I)$ where $\mathfrak{p}, \mathfrak{B}$, and $I$ are sets with $\mathfrak{p} \cap \mathfrak{B}=\varnothing$ and $I \subseteq \mathfrak{p} \times \mathfrak{B}$. The elements of $\mathfrak{p}$ are called points and those of $\mathfrak{B}$ are called blocks. If $(p, B) \in I$, we say $p$ and $B$ are incident. We usually write $p$ I $B$ or $B$ I $p$.

The dual structure $S^{*}=\left(\mathfrak{p}^{*}, \mathfrak{B}^{*}, I^{*}\right)$ of $S$ is defined by $\mathfrak{p}^{*}=\mathfrak{B}, \mathfrak{B}^{*}$ $=\mathfrak{p}$ and $(B, p) \in I^{*}$ if and only if $(p, B) \in I$.

For every $p \in \mathfrak{p}$, we denote by $[p]$ the number of blocks which are incident with $p$. For $p, q \in \mathfrak{p}$, we define $[\{p, q\}]$ to be the number of blocks incident with both $p$ and $q$. A partial plane is an incidence structure such that $[\{p, q\}] \leqq 1$ for distinct points $p$ and $q$.

An automorphism of an incidence structure $S=(\mathfrak{p}, \mathfrak{B}, I)$ is a bijection $\sigma: \mathfrak{p} \cup \mathfrak{B} \rightarrow \mathfrak{p} \cup \mathfrak{B}$ such that $p$ I $B$ if and only if $p \sigma$ I $B \sigma$ for all $p \in \mathfrak{p}$ and $B$

Presented to the Society, November 28, 1970; received by the editors March 15, 1974 and, in revised form, January 12, 1975.

AMS (MOS) subject classifications (1970). Primary 05B25, 05C25, 06A25, 20B25; Secondary 02J05, 06A40, 81A12.

Key words and phrases. Orthomodular lattices, automorphism groups, distributive lattices, graph theoretic methods, orthocomplemented lattices, Boolean lattices, graphs, finite incidence structures, orthogonality spaces, dual structures.

(c) American Mathematical Society 1976 
$\in \mathfrak{B}$. The set of automorphisms of $S$ forms a group which is denoted by $\operatorname{Aut}(S)$.

A graph $G$ consists of a finite nonempty set $V$ of points together with a prescribed set $X$ of unordered pairs of distinct points of $V$. Each pair $x=[u, v]$ of points in $X$ is a line of $G$. We say that $u$ and $v$ are adjacent points and that $u$ and $x$ are incident as are $v$ and $x$. A cycle of length $n$ in a graph is a sequence $\left(p_{0}, p_{1}, \ldots, p_{n}\right)$ of distinct points except that $p_{0}=p_{n}$ and such that $p_{i-1}$ and $p_{i}$ are adjacent $(i=1,2, \ldots, n)$. The girth of a graph $G$, denoted $g(G)$, is the length of a shortest cycle (if any) in $G$.

An automorphism of a graph $G$ is a bijection from the set of points of $G$ into itself which preserves adjacency. The group of automorphisms of $G$ is denoted by $\operatorname{Aut}(G)$.

An orthogonality graph is a pair $(X, \perp)$ where $\perp$ is a symmetric irreflexive relation on the set $X$.

A space is a pair $(X, \mathcal{E})$ where $X$ is a nonempty set and $\mathcal{E}$ is a family of nonempty subsets of $X$. A weight on a space $(X, \mathcal{E})$ is a mapping $m: X \rightarrow[0,1]$ such that $\sum_{a \in E} m(a)=1$ for all $E \in \mathcal{E}$. For any space $(X, \mathcal{E})$ and for each $x \in X$, define $\varepsilon_{x}=\{E: E \in \mathcal{E}$ and $x \in E\}$ and $\mathcal{E}(x)=\cup\left\{E: E \in \mathcal{E}_{x}\right\}$.

The dual space $\left(X^{*}, \mathcal{E}^{*}\right)$ of a space $(X, \mathcal{E})$ is defined by $X^{*}=\mathcal{E}$ and $\mathcal{E}^{*}$ $=\left\{\mathcal{E}_{x}: x \in X\right\}$. A space $(X, \mathcal{E})$ is an orthogonality space if

(1) $\cup\{E: E \in \varepsilon\}=X$,

(2) $E \in \mathcal{E}$ and $E \notin \mathcal{E}_{x}$ implies $E \nsubseteq \mathcal{E}(x)$,

(3) $M \subseteq X$ and $M \subseteq \cap\{\mathcal{E}(x): x \in M\}$ implies that $M \subseteq E$ for some $E \in \mathcal{E}$.

It is shown in [7] that every orthogonality graph $(X, \perp)$ determines a space $(X, \mathcal{E})$ and conversely. Thus let $\vartheta(X, \perp)$ denote the set of all $\perp$-sets of $(X, \mathcal{E})$. (Recall that $M \subseteq X$ is a $\perp$-set if $x, y \in M$ and $x \neq y$ imply $x \perp y$.) For each $M \subseteq X$ define $M^{\perp}=\{x \in X: x \perp m$ for all $m \in M\}$ and $M^{\perp \perp}$ $=\left(M^{\perp}\right)^{\perp}$. By the quasilogic of $(X, \mathcal{E})$ (as of $\left.(X, \perp)\right)$ we mean the set $\mathcal{E}=\left\{D^{\perp \perp}: D \in \mathcal{O}(X, \perp)\right\}$ partially ordered by set inclusion. We write $\mathcal{E}$ $=\mathfrak{L}(X, \mathcal{E})=\mathfrak{L}(X, \perp)$. A quasilogic $\mathcal{L}$ is a logic if $M \in \mathcal{L}$ implies $M^{\perp} \in \mathcal{L}$. An orthogonality space $(X, \mathcal{E})$ such that $\mathfrak{\varrho}(X, \mathcal{E})$ is a logic is called an orthocomplemented space. An orthogonality space $(X, \mathcal{E})$ is point closed if $\{x\}^{\perp \perp}$ $=\{x\}$ for all $x \in X$. We write $\{x\}^{\perp \perp}$ as $x^{\perp \perp}$.

By a state on a logic $\mathcal{E}=\mathcal{E}(X, \mathcal{E})$ we mean a mapping $\sigma: X \rightarrow[0,1]$ such that $\sigma(X)=1$ and if $M \subseteq N^{\perp}$, then $\sigma(M \vee N)=\sigma(M)+\sigma(N)$ for any $M$, $N \in \mathcal{L}$. A set $\mathcal{S}$ of states on $\mathcal{L}$ is full, if for $M, N \in \mathcal{L}, M \subseteq N$ if and only if $\sigma(M) \leqq \sigma(N)$ for all $\sigma \in \mathcal{S}$. A set $\mathcal{W}$ of weights on an orthogonality space $(X$, $\mathcal{E})$ is said to be full, if for any $x, y \in X, x \perp y$ if and only if $w(x)+w(y)$ $\leqq 1$ for all $w \in \mathscr{W}$. A pair $(\varrho, \delta)$ where $\delta$ is a full set of states on a logic $\varrho$ is called a quantum logic.

Define $(X, \mathcal{E})$ to be an $F$-space if every maximal scattered set intersects every maximal orthogonal set (a subset $S$ of $X$ is scattered if $x, y \in S$ implies $x$ $\notin y^{\perp}$ and a subset $T$ of $X$ is orthogonal if $\left.T \in \mathcal{O}(X, \perp)\right)$. Define $(X, \mathcal{E})$ to be a complete Dacey space if $K \subseteq X$ and $D$ a maximal orthogonal subset of $K^{\perp}$ implies $D^{\perp \perp}=K^{\perp}$.

Let $(X, \mathcal{E})$ be an orthogonality space. Choose an indexing set $I$ which is in one-to-one correspondence with $\mathcal{E}$ such that $I \cap X=\varnothing$ so that $\mathcal{E}=\left\{E_{i}: i\right.$ 
$\in I\}$. Let $Y=X \cup I$. Define $\sim$ on $Y$ by $x \sim y$ if and only if $x \perp y$ for $x, y \in X$ and $x \sim i$ if and only if $i \sim x$ if and only if $x \in E_{i}$ for $x \in X, i$ $\in I$. Then $(Y, \sim)$ is an orthogonality graph which determines an orthogonality space called the Dacification of $(X, \mathcal{E})$.

The automorphism group $\operatorname{Aut}(X, \mathcal{E})$ of $(X, \mathcal{E})$ is the automorphism group of its orthogonality graph.

Recall that a lattice is a set $L$ together with a partial ordering $\leqq$ on $L$ such that for any $x, y \in L$, the supremum and infimum of $x$ and $y$ (written $x \vee y$ and $x \wedge y$, respectively) both exist. An involution lattice is a lattice $(L, \leqq)$ in which there exists a map ': $L \rightarrow L$ such that

(1) $e \leqq f$ in $L$ implies $f^{\prime} \leqq e^{\prime}$,

(2) $e=e^{\prime \prime}$ for all $e \in L$. If furthermore $L$ is bounded and if for $e \in L, e^{\prime}$ is a complement of $e$, then $L$ is an orthocomplemented lattice.

An orthocomplemented lattice $\left(L, \leqq,^{\prime}\right)$ is an orthomodular lattice if for $e, f \in L, e \leqq f$, we have $f=e \vee\left(f \wedge e^{\prime}\right)$.

If $L$ is an orthomodular lattice, an automorphism of $L$ is a bijection from $L$ to $L$ which preserves $\vee, \wedge$, and '. The set of all automorphisms of $L$ is a group called the automorphism group of $L$ and is denoted by $\operatorname{Aut}(L)$.

We point out that, since the space $(X, \mathcal{E})$ is an incidence structure, we can consider the dual structure $(X, \mathcal{E})^{*}$ of $(X, \mathcal{E})$ and the dual space $\left(X^{*}, \mathcal{E}^{*}\right)$ of $(X$, $\mathcal{E})$. Note that since $\left(X^{*}, \mathcal{E}^{*}\right)$ is also a space, it too can be considered as an incidence structure. One naturally wonders when $(X, \mathcal{E})^{*} \cong\left(X^{*}, \mathcal{E}^{*}\right)$. This question is partially answered in 2.1 , the proof of which is straightforward. As in [7], we define the space $(X, \varepsilon)$ to be distinguishing if $\varepsilon_{x} \neq \varepsilon_{y}$ whenever $x \neq y$.

2.1 LeMMA. Let $(X, \mathcal{E})$ be any space.

(1) The space $\left(X^{*}, \mathcal{E}^{*}\right)$ is distinguishing.

(2) If $(X, \mathcal{E})$ is distinguishing, then $\left(X^{*}, \mathcal{E}^{*}\right) \cong(X, \mathcal{E})^{*}$ and, hence, $\left(X^{* *}, \mathcal{E}^{* *}\right)$ $\cong(X, \mathcal{E})^{* *}=(x, \mathcal{E})$

(3) If $(X, \mathcal{E})$ is a partial plane such that $[x] \geqq 2$ for all $x \in X$, then $(X, \mathcal{E})$ is distinguishing and, hence, $\left(X^{*}, \mathcal{E}^{*}\right) \cong(X, \mathcal{E})^{*}$ and $\left(X^{* *}, \mathcal{E}^{* *}\right) \cong(X, \mathcal{E})$.

In an incidence structure $S=(\mathfrak{p}, \mathfrak{B}, I)$, a loop of order $n$ is a chain $\left(x_{0}, x_{1}, x_{2}, \ldots, x_{2 n-1}, x_{0}\right)$ where $x_{2 k} \in \mathfrak{p}$ and $x_{2 k+1} \in \mathfrak{B}$ for $k=0,1,2, \ldots$, $n-1$, and where $x_{i} \neq x_{j}$ for $i, j=0,1,2, \ldots, 2 n-1$, and $i \neq j$.

2.2 Lemma. An orthocomplemented space $(X, \mathcal{E})$ is a complete Dacey space if and only if $\varrho(X, \mathcal{E})$ is an orthomodular lattice.

Proof. Straightforward.

2.3 Lemma. Let $S=(\mathfrak{p}, \mathfrak{B}, I)$ be an incidence structure. Then there exists a loop of order $n$ in $S$ if and only if there exists a loop of order $n$ in $S^{*}$.

Proof. If $\left(x_{0}, x_{1}, x_{2}, \ldots, x_{2 n-1}, x_{0}\right)$ is a loop of order $n$ in $S$, then $\left(x_{1}, x_{2}, x_{3}, \ldots, x_{2 n-1}, x_{0}, x_{1}\right)$ is a loop of order $n$ in $S^{*}$ and conversely.

2.4 Theorem. Let $G$ be a graph and $p \in G$.

(1) $G^{*}$ is a partial plane such that $[q]=2$ for all $q \in G^{*}$. 
(2) If $G$ has girth $g(G) \geqq 4$ and $[p] \geqq 2$, then $G^{*}$ is an orthogonality space.

(3) If $G$ has girth $g(G) \geqq 5$ and $\mid p] \geqq 3$, then $G^{*}$ is a point closed complete Dacey space.

Conversely assume $(X, \mathcal{E})$ is a finite space which is a partial plane such that $[x]=2$ for all $x \in X$. Then $(X, \mathscr{E})^{*} \cong\left(X^{*}, \mathcal{E}^{*}\right)$ and $(X, \mathcal{E})^{*}$ is a graph.

(4) If $(X, \mathcal{E})$ is an orthogonality space such that $|E| \geqq 2$ for all $E \in \mathcal{E}$, then $g\left((X, \mathscr{E})^{*}\right) \geqq 4$.

(5) If $(X, \mathcal{E})$ is a complete Dacey space such that $|E| \geqq 3$ for all $E \in \mathcal{E}$, then $g\left((X, \mathcal{E})^{*}\right) \geqq 5$.

Proof. (1) This follows directly from the definitions of graph, partial plane, and $[q]$.

(2) Now $G^{*} \neq \varnothing$ since $[p] \geqq 2$ for all $p \in G$. For each $a \in G$, let $A_{a}$ $=\left\{x: x \in G^{*}\right.$ and $\left.x \mathrm{I} a\right\}$. Now $A_{a} \neq \varnothing$ since $\left|A_{a}\right|=[a]=2$. Thus $\left(G^{*}, \delta\right)$ is a space where $\mathcal{E}=\left\{A_{a}: a \in G\right\}$. We show that the conditions of 1.3 of [7] are satisfied.

Now $E \in \mathcal{E}$ if and only if $E=A_{a}$ for some $a \in G$. Let $x \in G$. Since $[x]=2$, we have $x \mathrm{I} a$ for some $a \in G$ so that $x \in A_{a}$ for some $a \in G$. Hence $G^{*}=\cup\{E: E \in \mathcal{E}\}$.

Let $M, N \in \mathcal{E}$ and $M \neq N$. Then since $G^{*}$ is a partial plane, there exists $a, b \in G$ such that $a \neq b, M=A_{a}$ and $N=A_{b}$. Since $[a] \geqq 2$ there exists $x \in G^{*}$ such that $x \mathrm{I} a$. If $x X b$ we are through. If $x \mathrm{I} b$, there exists $y \in G^{*}$ such that $y \neq x$ and $y$ I $a$, since $[a] \geqq 2$. But $y X b$ since $G$ is a graph. Thus $M \nsubseteq N$.

Finally assume $M \subseteq G^{*}$ and for all $x, y \in M$ there is an $N_{x, y} \in \mathcal{E}$ such that $\{x, y\} \subseteq N_{x, y}$. We must show that there exists an $N \in \mathcal{E}$ such that $M \subseteq N$. If $|M| \leqq 2$, choose $N=N_{x, y}$. Let $|M| \geqq 3$. Fix $x, y \in M$ and suppose $M \nsubseteq N$ for all $N \in \mathcal{E}$. Then for every $N \in \mathcal{E}$ there exists $z \in M$ such that $z \notin N$. Hence $z \notin N_{x, y}$. By hypothesis there exist $N_{x, z}$ $\in \mathcal{E}$ and $N_{y, z} \in \mathcal{E}$ such that $\{x, z\} \subseteq N_{x, z}$ and $\{y, z\} \subseteq N_{y, z}$. Since $G^{*}$ is a partial plane, $N_{x, z} \neq N_{y, z}$. Thus $\left(x, N_{x, y}, y, N_{y, z}, z, N_{x, z}, x\right)$ is a loop of order three. This contradicts the girth condition of $G$.

(3) Now assume $G$ has girth $g(G) \geqq 5$ and $[p] \geqq 3$. Then by the above proof, $\left(G^{*}, \mathcal{E}\right)$ is an orthogonality space. We have $|M| \geqq 3$ for each $M$ $\in \mathcal{E}$ and $\left|M_{1} \cap M_{2}\right| \leqq 1$ for all $M_{1} \neq M_{2}$ where $M_{1}, M_{2} \in \mathcal{E}$. Thus, by 1.4 of [7] and Theorem 1 of Dacey [3], $\mathscr{\complement}\left(G^{*}, \mathcal{E}\right)$ is an orthocomplete orthomodular poset. By the Remark of Dacey [3], $\mathcal{L}\left(G^{*}, \mathcal{E}\right)$ satisfies Convention 1 of Greechie $[8]$ since $[p] \geqq 3$ and since $\left(G^{*}, \mathcal{E}\right)$ is a partial plane. Since $g(G) \geqq 5$, we have by 2.3 that $\left(G^{*}, \mathcal{E}\right)$ has no loops of order four. By the Remark of Dacey [3], $\mathcal{E}\left(G^{*}, \mathcal{E}\right)$ has no loops of order four and, hence, is an orthomodular lattice by the Atomistic Loop Lemma of Greechie [8]. By 2.2, $\left(G^{*}, \mathcal{E}\right)$ is a point closed complete Dacey space.

Conversely assume that $(X, \mathscr{E})$ is a space which is a partial plane such that $[x]=2$ for all $x \in X$. It is obvious that $(X, \delta)^{*}$ is a graph. We denote this graph by $H$. By (3) of 2.1 , we have that $\left(X^{*}, \varepsilon^{*}\right) \cong(X, \delta)^{*}$.

(4) Now assume that $(X, \mathcal{E})$ is also an orthogonality space such that $|E| \geqq 2$ for all $E \in \mathcal{E}$. Making repeated use of the fact that $|E| \geqq 2$ for all $E$ $\in \mathcal{E}$ and $[x]=2$ for all $x \in X$, it is easy to show that $(X, \mathscr{E})$ contains a loop 
and, hence, that $H$ contains a cycle by 2.3 . No graph can contain a 1-cycle or a 2-cycle. Thus suppose $H$ contains a 3 -cycle, say $C=(a, x, b, y, c, z, a)$ so that all the elements of $C$ are distinct and $x X c, y X a$, and $z X b$. Hence, in $(X, \mathcal{E})$ there exists no $N \in \mathcal{E}$ such that $M=\{x, y, z\} \subseteq N$. But any pair of points of $M$ are in a block. This contradicts condition (3) of 1.3 of [7].

(5) Finally assume $(X, \mathcal{E})$ is a complete Dacey space such that $|E|$ $\geqq 3$ for all $E \in \mathcal{E}$. Then since $[x]=2$ for all $x \in X$, we have that $\mathcal{L}(X, \mathcal{E})$ satisfies Convention 1 of Greechie [8]. Hence, every loop of $(X, \mathcal{E})$ is of order $\geqq 5$. This says that $g(H) \geqq 5$.

3. The main result. Using the techniques of Frucht [5], the following result can be easily proven.

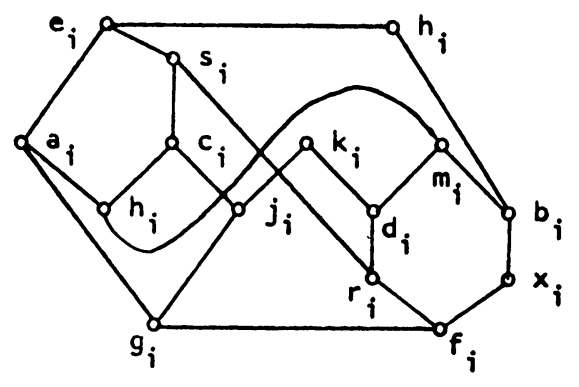

FIGURE 1

3.1 Lemma. The graph $G_{i}$ given in Figure 1 has girth five and the identity permutation as its automorphism group.

3.2 LemMA. Let $L$ be any atomic orthocomplete orthomodular lattice. Then $\operatorname{Aut}(L) \cong \operatorname{Aut}(A, \perp)$ where $A$ is the set of atoms of $L$.

Proof. Let $\sigma \in \operatorname{Aut}(A, \perp)$ and $a \in A$. For $x \in L$, define $\sigma^{\prime}(x)=$ $\bigvee_{a \leqq x} \sigma(a)$, which exists since $L$ is orthocomplete. Using the fact that $A$ is joindense as in [3], it is routine to show that $\sigma^{\prime} \in \operatorname{Aut}(L)$ and $\sigma \rightarrow \sigma^{\prime}$ is a group isomorphism.

3.3 Lemma. Let $(X, \mathcal{E})$ be a point closed complete Dacey space. Then $\operatorname{Aut}(X, \mathcal{E}) \cong \operatorname{Aut}(\mathscr{E}(X, \mathcal{E}))$.

Proof. By $2.2, \mathfrak{E}(X, \mathcal{E})$ is an orthocomplete orthomodular lattice. It is also atomic and by the Remark of Dacey [3], $(X, \mathcal{E}) \cong(A, \perp)$ where $A$ is the set of atoms of $\mathcal{L}(X, \mathcal{E})$. By 3.2 we have $\operatorname{Aut}(X, \mathcal{E}) \cong \operatorname{Aut}(A, \perp) \cong \operatorname{Aut}(\mathcal{L}(X, \mathcal{E}))$.

3.4 THEOREM. Let $H$ be a finite group. Then there exists a finite atomic orthomodular lattice $L$ such that $\operatorname{Aut}(L) \cong H$.

Proof. If $H$ is the identity permutation $I$, let $L=\{0,1\}$. Thus suppose $H \neq I$. By 1.2 of Sabidussi [13], there exists a regular graph $F_{1}$ of degree five such that $\operatorname{Aut}\left(F_{1}\right) \cong H$. Suppose $F_{1}$ has $q$ lines. From $F_{1}$ we construct the subdivision graph $F_{2}$ as follows. For every line $[u, v]_{i}$ of $F_{1}$, add a point $x_{i}$ and replace the line $[u, v]_{i}$ by the two lines $\left[u, x_{i}\right]$ and $\left[v, x_{i}\right]$ where $i=1,2, \ldots, q$. It is apparent that $\operatorname{Aut}\left(F_{1}\right) \cong \operatorname{Aut}\left(F_{2}\right)$. 
From $F_{2}$ construct another graph $G$ as follows. For $i=1,2, \ldots, q$, we attach the graph $G_{i}$ to $F_{2}$ by identifying the point $x_{i}$ of $G_{i}$ with the point $x_{i}$ of $F_{2}$.

Let

$$
\begin{aligned}
A & =\left\{x: x \text { is a point of } F_{2} \text { which was orginally in } F_{1}\right\}, \\
B & =\left\{x: x=x_{i}\right\}, \text { and } \\
W_{i} & =\left\{x: x \text { is a point of } G_{i} /\left\{x_{i}\right\}\right\} .
\end{aligned}
$$

Note that $\operatorname{deg}(x)=5$ if $x \in A, \operatorname{deg}(x)=4$ if $x \in B$ and $\operatorname{deg}(x)=3$ if $x$ $\in W_{i}$. Note also that $g(G)=5$ since $g\left(G_{i}\right)=5$ and $g\left(F_{2}\right) \geqq 6$.

Let $\sigma \in \operatorname{Aut}(G)$. By 3.1, no points of $W_{i}$ are permuted among themselves by $\sigma$. Consideration of the degrees of points of $G$ shows that $\sigma(A)=A, \sigma(B)$ $=B$, and $\sigma\left(W_{i}\right)=W_{j}$ if and only if $\sigma\left(x_{i}\right)=x_{j}$ in which case each point of $W_{i}$ is taken by $\sigma$ into the corresponding point of $W_{j}$ where $i, j=1,2, \ldots, q$ (viz. $\sigma\left(a_{i}\right)=a_{j}$, etc.). Thus Aut $(G) \cong \operatorname{Aut}\left(F_{2}\right)$. By Theorem 14.1 of Harary [9], we have Aut $\left(G^{*}\right) \cong$ Aut $(G)$ and by $2.4(3)$, we have that $G^{*}$ is a point closed complete Dacey space. By 3.3 , we have Aut $\left(\mathcal{L}\left(G^{*}\right)\right) \cong \operatorname{Aut}\left(G^{*}\right)$. Collecting our results we have

$$
\operatorname{Aut}\left(\mathfrak{L}\left(G^{*}\right)\right) \cong \operatorname{Aut}\left(G^{*}\right) \cong \operatorname{Aut}(G) \cong \operatorname{Aut}\left(F_{2}\right) \cong \operatorname{Aut}\left(F_{1}\right) \cong H \text {. }
$$

Since $\mathfrak{Q}\left(G^{*}\right)$ is an orthomodular lattice by 2.2 , the theorem is proved.

3.5 Corollary. Let $H$ be a finite group. Then there exists a finite quantum logic $Q$ such that $\operatorname{Aut}(Q) \cong H$.

Proof. Let $G^{*}$ be as in 3.4. Let $D\left(G^{*}\right)$ be the Dacification of $G^{*}$. Clearly $\operatorname{Aut}\left(D\left(G^{*}\right)\right) \cong \operatorname{Aut}\left(G^{*}\right)$. Now the point closed complete Dacey space $D\left(G^{*}\right)$ is also an $F$-space, being the Dacification of a complete Dacey space and, hence, admits a full set of weights. By 1.6 of [7], $\varrho\left(D\left(G^{*}\right)\right)$ admits a full set of states. Thus $\mathcal{L}\left(D\left(G^{*}\right)\right)$ is a quantum logic. By $3.3, \operatorname{Aut}\left(D\left(G^{*}\right)\right)$ $\cong \operatorname{Aut}\left(\mathcal{L}\left(D\left(G^{*}\right)\right)\right)$ and the result obtains.

As a final remark, we observe that $L$ is not necessarily unique in 3.4 as can be seen by observing that $\operatorname{Aut}\left(G_{32}\right) \cong \operatorname{Aut}\left(2^{5}\right) \cong S_{5}$. Here $S_{5}$ is the group of all permutations on five elements, $2^{5}$ is the orthomodular lattice of all subsets of a five element set, and $G_{32}$ is the well-known orthomodular lattice of Greechie [8].

\section{BIBLIOGRAPHY}

1. G. Birkhoff, On groups of automorphisms, Rev. Un. Mat. Argentina 11 (1946), 155-157. (Spanish) MR 7, 411.

2. G. Birkhoff and J. von Neumann, The logic of quantum mechanics, Ann. of Math. 37(1936), 823-843.

3. J. C. Dacey, Orthomodular spaces and additive measurement, Caribbean J. Sci. and Math. 1 (1969), 51-66. 
4. P. Dembowski, Finite geometries, Ergebnisse der Mathematik und ihrer Grenzgebiete, Band 44, Springer-Verlag, Berlin and New York, 1968. MR 38 \# 1597.

5. R. Frucht, Graphs of degree three with a given abstract group, Canad. J. Math. 1 (1949), 365-378. MR 11, 377.

6. - Lattices with a given abstract group of automorphisms, Canad. J. Math. 2 (1950), 417-419. MR 12, 473.

7. E. R. Gerelle, R. J. Greechie and F. R. Miller, Weights on spaces, Physical Reality and Mathematical Description, edited by C. P. Enz and J. Mehra, Reidel, Dordrecht, 1974, pp. 169 ff.

8. R. J. Greechie, Orthomodular lattices admitting no states, J. Combinatorial Theory 10 (1971), no. 2, 119-132.

9. F. Harary, Graph theory, Addison-Wesley, Reading, Mass., 1969. MR 41 \# 1566.

10. G. W. Mackey, The mathematical foundations of quantum mechanics: A lecture-note volume, Benjamin, New York, 1963. MR 27 \#5501.

11. C. H. Randall, $A$ mathematical foundation for empirical science with special reference to quantum theory. I: A calculus of experimental propositions, Knolls Atomic Power Labs., Report KAPL-3147, June, 1966.

12. C. H. Randall and D. J. Foulis, An approach to empirical logic, Amer. Math. Monthly 77 (1970), 363-374. MR 41 \#3334.

13. G. Sabidussi, Graphs with given group and given graph-theoretical properties, Canad. J. Math. 9 (1957), 515-525. MR 20 \#1322.

Department of Mathematics, Central Missouri State University, Warrensburg, MisSOURI 64093 